\title{
DOS ESPECIES NUEVAS DE PLEUROTHALLIDINAE (ORCHIDACEAE) DE MÉXICO
}

\author{
Rodolfo Solano Gómez
}

\begin{abstract}
Centro Interdisciplinario de Investigación para el Desarrollo Integral Regional, Unidad Oaxaca, Instituto Politécnico Nacional. Hornos 1003, Santa Cruz Xoxocotlan, 71230, Oaxaca, México. solanogo@yahoo.com.mx.
\end{abstract}

\begin{abstract}
Resumen: Se describen e ilustran dos especies nuevas de la subtribu Pleurothallidinae como Acianthera sotoana, de Oaxaca y Veracruz, y Stelis sotoarenasii, de Oaxaca. Estas orquídeas habían sido confundidas previamente con especies similares, la primera con Acianthera johnsonii, la segunda con Stelis retusa. Para cada especie se proporciona información sobre distribución, hábitat, fenología, estado de conservación y un mapa de sus localidades conocidas.
\end{abstract}

Aвstract: Two new species of subtribe Pleurothallidinae are described and illustrated as Acianthera sotoana, from Oaxaca and Veracruz, and Stelis sotoarenasii, from Oaxaca. These orchids have been previously confused with similar species, the former with Acianthera johnsonii, the latter with Stelis retusa. For each species information about distribution, habitat, phenology, conservation status, and a map with their known localities are provided.

Palabras clave / Key words: Acianthera sotoana, endemismos, Oaxaca, orquídeas, Stelis sotoarenasii, Veracruz

En los últimos años, la revisión de las especies de la subtribu Pleurothallidinae para México ha hecho que éste sea uno de los grupos mejor estudiados de la orquideoflora nacional (Soto Arenas, 1987; Hágsater \& Salazar, 1990; Solano Gómez 1993, 1999, 2000; Salazar \& Soto Arenas, 1996; Hágsater \& Soto Arenas, 2003; García-Cruz et al., 2003; LópezVelázquez et al., 2007; Solano Gómez \& Salazar, 2007; Solano Gómez et al., 2008; Hágsater \& Soto Arenas, 2008). La diversidad de este grupo en México está representada por 20 géneros, 203 especies y 3 taxones infraespecíficos (Soto Arenas et al., 2007). Si bien para la mayoría de las especies su delimitación taxonómica ha quedado bien establecida, permanecen varias que no han sido publicadas, por lo que en este trabajo dos de ellas son publicados como nuevas a partir de material vivo y herborizado; cada especie se acompaña de un dibujo a línea detallado y se proporciona un mapa para indicar su distribución conocida en México.

Acianthera sotoana R.Solano, sp. nov.

TIPO: MÉXICO. Oaxaca: Municipio Totontepec Villa de
Morelos, cañón del Río Toro, sobre el banco del río, $1800 \mathrm{~m}$, colectado septiembre 1977, prensado 23 julio 1983, O. Suárez 566 (holotipo, AMO; isotipo, AMO). FIG. 1, 3.

Acianthera johnsonii (Ames) Pridgeon \& M.W.Chase affinis autem laciniate-calyce pubescenti extus et intus, petalis non falcatibus, labello acuto ad apicem, et habitu differt.

Hierba rupícola, a veces epífita, cortamente rizomatosa, hasta de $36.5 \mathrm{~cm}$ de altura. Raices blanquecinas, flexuosas, de 1.2-2.0 $\mathrm{mm}$ de diámetro. Rizoma muy corto, engrosado, de 8-10 mm de longitud entre tallos adyacentes, $4.5-6.0 \mathrm{~mm}$ de grosor. Tallos erectos, cilíndricos, formados por 4-5 entrenudos, 4.0$17.5 \mathrm{~cm}$ de largo, $1.6-3.5 \mathrm{~mm}$ de diámetro; cubiertos totalmente por vainas lateralmente comprimidas, infladas hacia el ápice, imbricadas, obtusas, carinadas a lo largo de las nervaduras, caedizas, densamente manchadas de púrpura. Hoja sésiles, una por tallo, apical, sésil, elíptica, obtusa, coriácea, cortamente bilobada y mucronada en el ápice, ligeramente recurvada en los márgenes, 10-19 x 4-6 cm. 
Inflorescencia racemosa, generalmente de la base del tallo y colgante, a veces también del ápice del tallo, erecta y reclinada sobre la superficie interna de la hoja, 5.0-6.5 cm de largo, con hasta 10 flores que abren simultáneamente, inflorescencia basal ligeramente más larga que la apical; pedúnculo cilíndrico, $1-3 \mathrm{~cm}$ de largo, $2 \mathrm{~mm}$ de diámetro, en la inflorescencia apical rodeado por una bráctea espatácea, conduplicada, triangular, obtusa, escariosa, caediza; brácteas del pedúnculo 2 en la inflorescencia apical, hasta 5 en la inflorescencia basal, oblicuamente infundibuliformes, imbricadas, obtusas, membranáceas, densamente cubiertas de manchas púrpura, 6.0-7.7 cm de largo. Brácteas florales similares a las del pedúnculo pero más grandes, 7.9-8.7 $\mathrm{mm}$ de largo. Ovario subpiramidal, subtriangular en sección transversal, engrosado hacia el ápice, verde con manchas púrpura, glandularpubescente; articulado en un ángulo casi recto a un pedicelo cilíndrico. Flores casi perpendiculares al racimo, poco abiertas, $17-21 \mathrm{~mm}$ de largo; sépalos café-anaranjados o amarillo-anaranjados, densamente manchados de púrpura por ambas superficies; pétalos café-anaranjados con manchas púrpura; labelo anaranjado con manchas púrpura; columna y antera púrpura. Sépalos casi paralelos a la columna, carnosos, mucronados en el ápice, glandular-pubescentes en ambas superficies, los pelos rojo muy oscuro, ciliados en los márgenes; sépalo dorsal lanceolado a oblongolanceolado, subagudo, 7-nervado, carinado sobre la nervadura media, 17.0-18.5 × $5 \mathrm{~mm}$; sépalos laterales unidos entre sí por dos tercios de su longitud para formar un sinsépalo cóncavo, carnoso, oblongolanceolado, bipartido, 18.4-21.5 x 10.0-13.4 mm, cada sépalo 7-nervado, carinado sobre la nervadura media. Pétalos paralelos a la columna, cartilaginosos, lanceolado-subrómbicos, oblicuos, acuminados, angulados en la mitad, ciliados en los dos tercios superiores, 3-nervados, 11.2-12.3 x $2.0 \mathrm{~mm}$. Labelo provisto de una uña trapezoidal que se une al pie de columna, en posición natural paralelo con respecto a la columna, arqueado, carnoso, conduplicado, papiloso en la superficie interna, 3-nervado, $5.5 \times 2.1 \mathrm{~mm}$; cuando extendido cordado-sagitado, subagudo, 6.7 x $3.7 \mathrm{~mm}$; con dos callos engrosados, subparalelos y ligeramente erectos sobre la lamina, más un callo prominente, rojizo e incurvado hacia la base. Columna delgada, ligeramente arqueada, el cuerpo de $5 \mathrm{~mm}$ de largo y $1 \mathrm{~mm}$ de ancho a nivel del estigma; con un pie de columna bien desarrollado, incurvado, casi la mitad de largo que el cuerpo de la columna; clinandrio cubriendo totalmente la antera, 3-dentado. Estigma formado por una cavidad en la superficie ventral de la columna; rostelo ventral, laminar, convexo. Antera ventral, esférico-ovoide, diminutamente glandulosa en la superficie ventral, $1 \mathrm{~mm}$ de largo, $0.8 \mathrm{~mm}$ de ancho. Polinario formado por dos polinios ovoides, lateralmente comprimidos, amarillos, $0.6 \mathrm{~mm}$ de largo, unidos por caudículas granulosas.

Otros especímenes: México. Oaxaca: Municipio Totontepec Villa de Morelos, cañón del Río Toro, sobre el banco del río, $1800 \mathrm{~m}$, septiembre 1977, prensado 20 junio 1981, O. Suárez 566 (AMO). Municipio Totontepec Villa de Morelos, cañón del Río Toro, sobre el banco del río, 1800 m, septiembre 1977, Greenwood s.n. (AMO). Municipio Totontepec Villa de Morelos, cañón del Río Toro, sobre el banco del río, $1800 \mathrm{~m}$, septiembre 1977, O. Suárez 2500 (OAX). Municipio Totontepec Villa de Morelos, Santa María Huitepec, río Llano, bosque de galería con Platanus rodeado de selva mediana perennifolia, $1497 \mathrm{~m}, 17^{\circ} 12^{\prime} 8.27^{\prime} \mathrm{N}$, $96^{\circ} 1$ '28.35\%O, rupícola, abundante a la orilla del río, 13 marzo 2009, R. Solano \& A. Martínez s.n. (OAX). Veracruz: Municipio Pajapan, cima del volcán San Martín Pajapan, bosque mesófilo de montaña, 1125 $\mathrm{m}, 18^{\circ} 18^{\prime} \mathrm{N}, 94^{\circ} 44^{\prime} \mathrm{O}$, epífita, rara, flores anaranjadas con puntos morados, 2 septiembre 2007, T. Krömer \& E. Otto 2954 (MEXU). Municipio de Tatahuicapan de Juárez, faldas del volcán Santa Martha, bosque mesófilo de montaña, $1500 \mathrm{~m}, 18^{\circ} 19^{\prime} \mathrm{N}, 94^{\circ} 50^{\prime} \mathrm{O}$, epífita, rara, flores amarillas con manchas moradas, 28 agosto 2008, T. Krömer 3696 (MEXU). Municipio Ixhuacán de los Reyes, Comapa, bosque mesófilo de montaña, $1560 \mathrm{~m}, 19^{\circ} 18^{\prime} 30^{\prime} \mathrm{N}, 97^{\circ} 5^{\prime} 10^{\prime} \mathrm{O}$, rupícola, regular, con cápsulas, 19 octubre 2008, J. Viccón \& M. Castañeda 199 (MEXU).

Distribución: Esta especie es endémica de México y hasta ahora solo ha sido registrada en montañas de la vertiente del Golfo de México (Fig. 3), en el Eje Volcánico Transversal y la Sierra Madre Oriental.

HÁBItAT: : Las poblaciones de esta especie se localizan entre 1125 y $1800 \mathrm{~m}$ de altitud, en zonas con selva mediana perennifolia, con presencia de arroyos permanentes y neblinas frecuentes durante todo el 


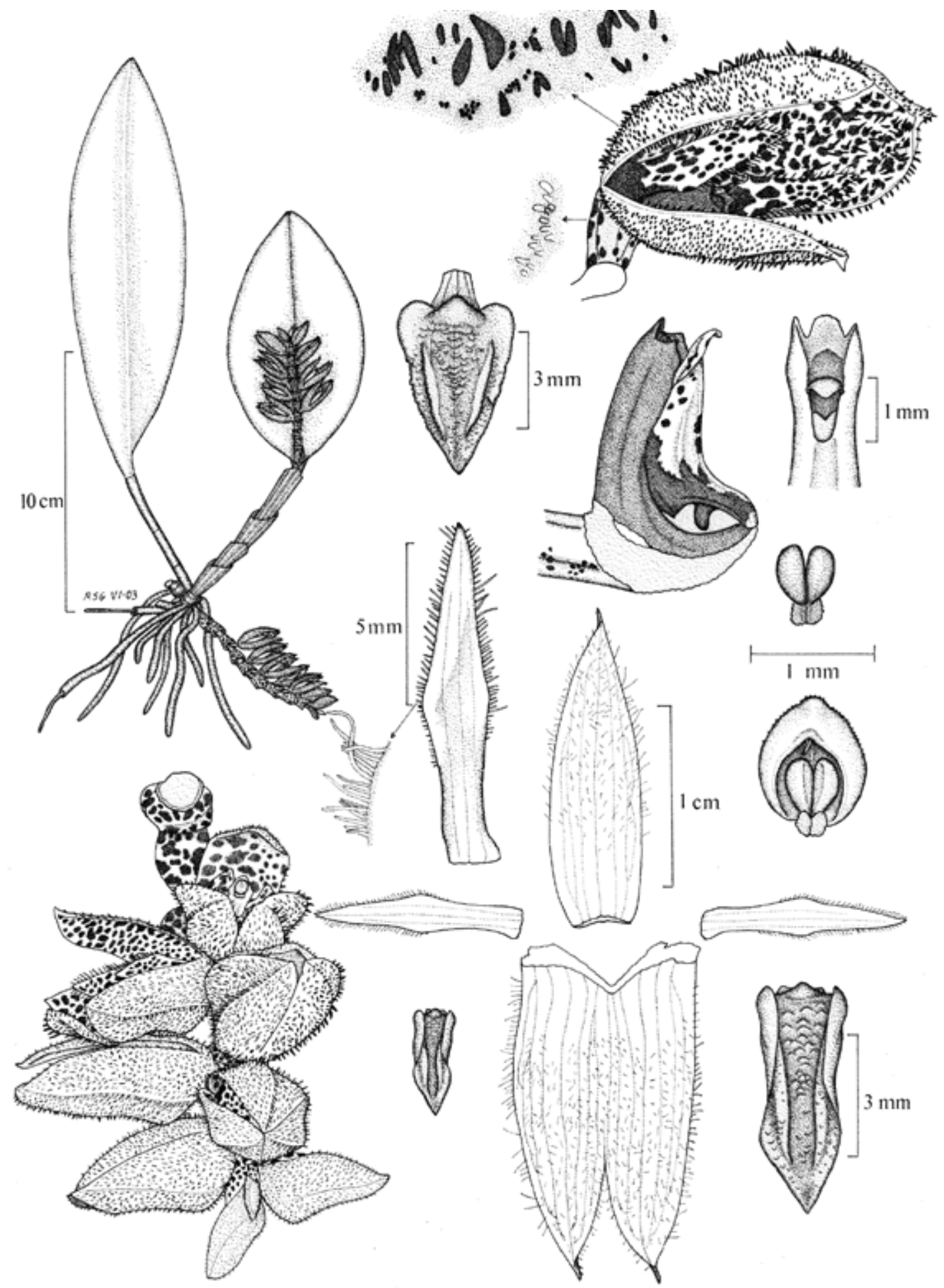

Figura 1. Acianthera sotoana R.Solano, basado en O. Suárez 566, (AMO, holo.), dibujo de R. Solano. 
año. Las plantas crecen generalmente sobre rocas encima de una capa de musgo o materia orgánica, pero a veces también llegan a establecerse como epífitas. Las localidades de esta especie se conocen en zonas de clima semicálido húmedo, con un régimen de lluvias en verano, temperatura media anual de $18^{\circ} \mathrm{C}$ y precipitación superior a los $2000 \mathrm{~mm}$ al año.

Fenología: Esta especie florece entre los meses de junio y septiembre, ha sido colectada con frutos en desarrollo en octubre y se han visto frutos secos poco después de haber liberado las semillas en marzo, época en la que empieza el crecimiento vegetativo.

EsTADO DE CONSERVACIÓN. No evaluado. Las poblaciones conocidas de esta especie se presentan dentro o en las inmediaciones de áreas naturales protegidas: el Parque Nacional Pico de Orizaba-Cofre de Perote (Veracruz), Reserva de la Biosfera Los Tuxtlas (Veracruz) y Reserva Comunal de Santa María Huitepec (Oaxaca), donde aún existe hábitat favorable para la orquídea.

Etimología: Esta especie está dedicada a Miguel Ángel Soto Arenas, un maestro, amigo y colega para la mayoría de orquideologos mexicanos en activo, por su invaluable aportación al estudio de las orquídeas mexicanas.

Esta especie, junto con A. herrerae (Luer) R.Solano $\&$ Soto Arenas y A. johnsonii forman un grupo que se caracteriza porque la inflorescencia surge de la base del tallo y de su ápice, el cual recientemente fue incluido, al igual que las especies de Echinosepala Pridgeon \& M.W.Chase, en el género Brenesia Schltr. (Luer, 2004). Aunque los primeros ejemplares de Acianthera sotoana fueron colectados desde 1977, éstos fueron confundidos con A. johnsonii, una especie centroamericana que en México se conoce de la región de los Altos de Chiapas; sin embargo, la segunda es diferente por sus plantas de menor tamaño, flores más pequeñas, sépalos laterales más elípticos, pétalos más arqueados y el labelo con el ápice redondeado y el callo basal menos erecto (Solano Gómez \& Soto Arenas, 2003). Por su parte, A. herrerae tiene sépalos con el mucrón más largo, pétalos abruptamente acuminados $\mathrm{y}$ atenuados, labelo con una forma muy diferente y provisto de lóbulos laterales uncinados (Luer, 1991, 2004). Previamente se había usado el nombre inédito "Acianthera mixe" para la especie aquí descrita y así había sido registrada por algunos autores (Soto Arenas et al., 2007; Solano Gómez et al., 2008).

Stelis sotoarenasii R.Solano, sp. nov.

TIPO: MÉXICo. Oaxaca: Municipio Villa Sola de Vega, La Cumbre, carretera Oaxaca-Puerto Escondido, cerca de las torres de microondas, $2179 \mathrm{~m}, 16^{\circ} 27^{\prime} 12.32^{\prime \prime} \mathrm{N}$, $97^{\circ} 0$ ' 12.2'” $\mathrm{O}$, bosque de pino-encino, epífita en encino, abundante, 26 abril 2009, R. Solano 2550 (holotipo, OAX; isotipos, AMO, MEXU). FIg. 2, 3.

Stelis retusa (Ames) Pridgeon \& M.W.Chase similaris sed folio latior ad apicem rotundato, racemis laxis, et floribus majoribus differt.

Hierba epifita, cespitosa, hasta de $14 \mathrm{~cm}$ de alto. Raices 0.7-1.2 mm grosor. Tallos formados por 2 entrenudos, 2-6 cm largo, 1.0-1.5 mm diámetro, cubiertos totalmente por vainas tubulares, obtusas, carinadas y mucronadas, escariosas, caedizas; con un anillo engrosado cerca del ápice. Hoja una por tallo, apical, carnosa, erecta, oblanceolada, redondeada, ligeramente recurvada en el ápice, 3.5-9.5 x 1.3$1.8 \mathrm{~cm}$, la base subpeciolada, atenuada, acanalada.. Inflorescencia surgiendo del anillo del tallo, racemosa, más corta que la hoja, reclinada sobre la superficie foliar interna, $3-5 \mathrm{~cm}$ largo, con 4 a 8 flores que abren sucesivamente; pedúnculo, cilíndrico, 1.3$2.3 \mathrm{~cm}$ de largo, rodeado en la base por una bráctea espatácea, conduplicada, triangular, obtusa, escariosa; brácteas del pedúnculo 2 , la basal cubierta por la bráctea espatácea, tubulares, oblicuas y obtusas en el ápice, membranáceas. Brácteas florales tubularinfundibuliformes, obtusas, cortamente apiculadas, membranáceas, 3.5-5.0 mm de largo. Ovario arqueado, ensanchado hacia el ápice, subtriangular en sección transversal, 4.3-4.9 mm largo; articulado a un pedicelo de 3.6-7.0 mm de largo. Flores bilabiadas, ligeramente colgantes, 9.0-9.5 mm de largo (de la base al ápice de los sépalos), 7.3-9.2 $\mathrm{mm}$ de apertura (entre el ápice del sépalo dorsal y los ápices de los laterales), 3.0-3.7 mm de ancho (entre los pétalos); los segmentos amarillos, densamente rayados y manchados de púrpura oscuro, columna blanca con manchas púrpura, antera amarilla con un mancha apical púrpura. Sépalos carnosos, 3-nervados; sépalo dorsal oblongo-lanceolado, obtuso, recurvado en el ápice, quillado en la superficie externa 


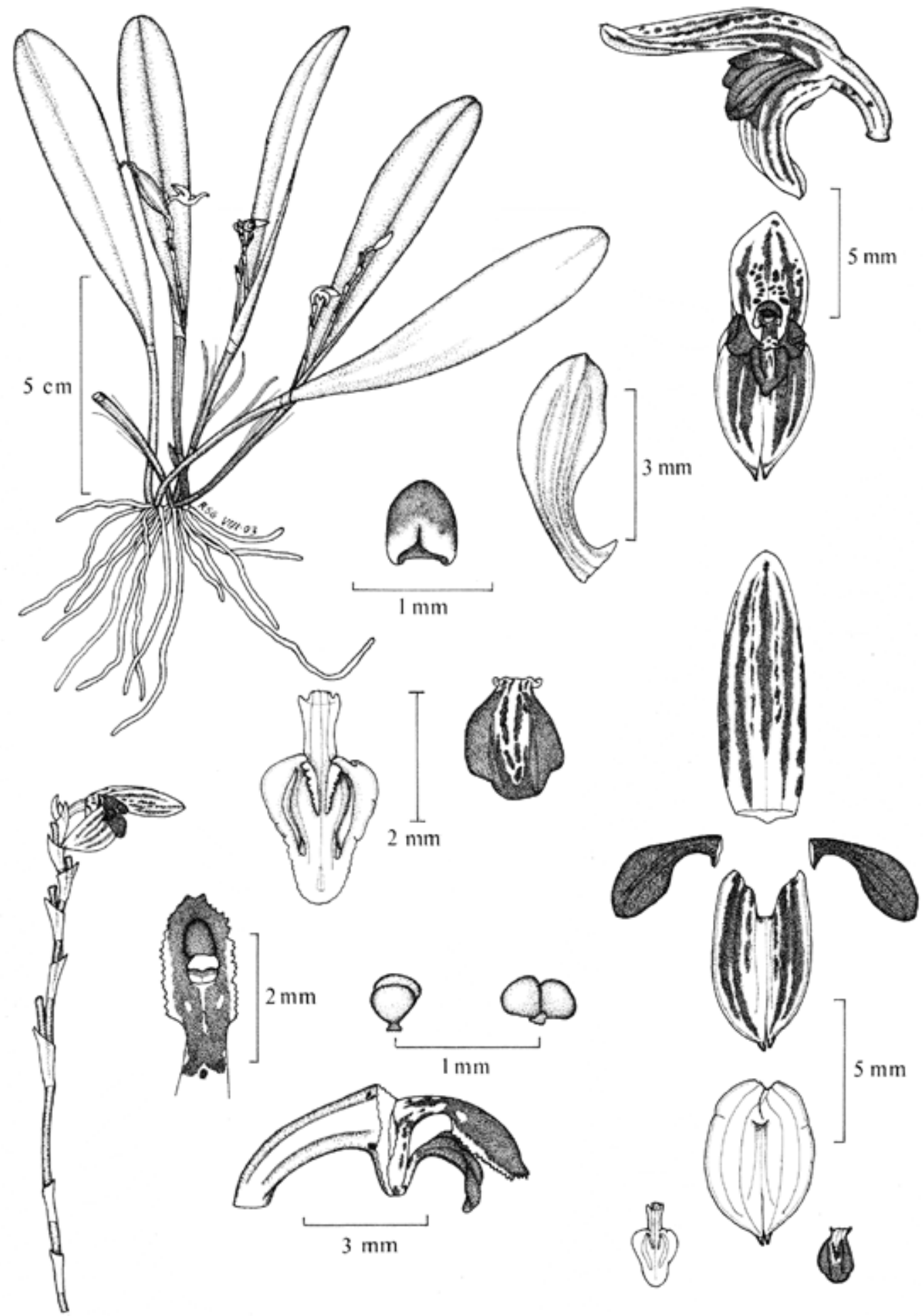

Figura 2. Stelis sotoarenasii R.Solano, basado en O. Suarez 2014, (AMO), dibujo de R. Solano. 
a lo largo de la nervadura media, 9.0-9.4 x 3.0-3.4 $\mathrm{mm}$; sépalos laterales connados por casi dos tercios de su longitud en un sinsépalo arqueado-recurvado, conduplicado, cortamente bilobado en el ápice, con la porciones libres adherentes, cuando extendido formando una lamina oval, obtusa, 5.5-6.0 x 3.3-3.5 $\mathrm{mm}$; el ápice de cada sépalo cortamente apiculado. Pétalos erectos, cartilaginosos, falcado-espatulados, subredondeados, abruptamente atenuados en la mitad basal, 3-nervados, carinados en la superficie externa a lo largo de las nervaduras, $4.0-4.3 \times 1.5-2.0 \mathrm{~mm}$. Labelo provisto de una uña basal que se une al pie de columna, carnoso, arqueado-recurvado, conduplicado, 3-nervado, 1.8-2.0 x 1.3-1.5 mm en posición natural; la uña oblonga, 2-auriculada en la base, $1.0 \times 0.6-0.8 \mathrm{~mm}$; la lamina extendida ligeramente 3 -lobada, obovadasubcordiforme, redondeada, $2.0-2.2 \times 1.7 \mathrm{~mm}$; con dos quillas erectas, prominentes que siguen el contorno de los márgenes y se extienden en los dos tercios basales de la lámina, mas otros dos callos internos más pequeños, crestados, lamelados, extendidos en el tercio basal de la lámina, aproximados en el ápice y divergentes hacia la base. Columna con un cuerpo delgado, fuertemente arqueado, alado, 3.5-3.6 mm de largo, $1 \mathrm{~mm}$ de ancho; provista de un pie prominente, descendente, ligeramente más corto que el cuerpo; clinandrio cubriendo totalmente la antera, con el margen denticulado; alas prominentes, proyectadas hacia el labelo, con el margen denticulado. Estigma ventral, cóncavo, subcuadrado, cubierto por una sustancia viscosa y transparente; rostelo ventral, laminar, convexo. Antera ventral, ovoide, $0.6 \times 0.4$ $\mathrm{mm}$. Polinario formado por 2 polinios piriformes, lateralmente comprimidos, $0.35 \mathrm{~mm}$ de largo, provistos de caudículas granulosas.

Otros especímenes: México. Oaxaca: Municipio Villa Sola de Vega, La Cumbre, carretera Oaxaca-Puerto Escondido, cerca de las torres de microondas, $2179 \mathrm{~m}$, $16^{\circ} 27^{\prime} 12.32^{\prime}$ 'N, $97^{\circ} 0$ ' $12.2^{\prime}$ 'O, bosque de pino-encino, epífita en encino, G. Cruz s.n. (OAX). Abundante. Municipio San Juan Tepeuxila, camino CuicatlánTlacolula, casi $4 \mathrm{~km}$ después de Tepeuxila, $2100 \mathrm{~m}$, $17^{\circ} 43^{\prime} 47^{\prime} \mathrm{N}, 96^{\circ} 49^{\prime} 07^{\prime} \mathrm{W}$, bosque de encino, epífita, 10 julio 2004, R. Jiménez 2451, G. Salazar y J. Reyes (AMO). Municipio Oaxaca de Juárez, Sierra de San Felipe, $17^{\circ} 4^{\prime} \mathrm{N}, 96^{\circ} 43^{\prime} \mathrm{O}$, bosque de pino-encino, $O$.
Suárez s.n. (OAX). Municipio Villa Sola de Vega, km 90 de la carretera Oaxaca-Puerto Escondido, O. Suárez 2014, AMO. Municipio Villa Sola de Vega, km 114.7 de la carretera Oaxaca-Puerto Escondido, 2 noviembre 1974, E. Greenwood s.n. [AMO (diapositiva)].

Distribución. Esta especie es endémica de México y hasta ahora solo se conoce de las vertientes interiores de las Sierra Madre Oriental y la Sierra Madre del Sur en Oaxaca (FIg. 3).

HÁbitat: Esta especie se encuentra entre 1500 y 2200 m de elevación, en bosques de encino y pinoencino con una estación de sequía bien marcada. Las plantas crecen como epífitas en troncos y ramas gruesas de encinos, directamente sobre la corteza o sobre una capa de musgo. Prospera en zonas de clima templado subhúmedo, donde las corrientes de viento son constantes y eso hace que no sean tan húmedos al interior.

Fenología. En campo se han visto ejemplares de esta especie con flores desde abril, en cultivo la floración se extiende hasta octubre. No se han observado ejemplares con frutos desarrollados.

Estatus de Conservación. No evaluado. Aunque solo una de sus localidades se presenta en un área protegida, la Reserva de la Biosfera Tehuacán-Cuicatlán, en las otras el hábitat es aún favorable y sus poblaciones pueden llegar a ser relativamente abundantes, formando en un solo árbol hospedero colonias integradas por hasta algunas decenas de individuos.

Etimología. El nombre de esta especie honra a Miguel Ángel Soto Arenas por su excelente contribución al estudio de la taxonomía y conservación de las orquídeas mexicanas.

En el pasado esta especie ha sido confundida con Stelis retusa, considerándola como una forma geográficamente aislada de ésta. Sin embargo, S. retusa difiere en sus plantas de mayor tamaño, con hojas más angostas, racimo relativamente más florífero y los segmentos florales ligeramente más pequeños; se trata de una orquídea común en las montañas del centro y occidente de México, en el Eje Volcánico Transversal, mientras que S. sotoarenasiii presenta una distribución restringida a Oaxaca. Otra especie muy similar es $S$. aristocratica, pero en esta las plantas son 


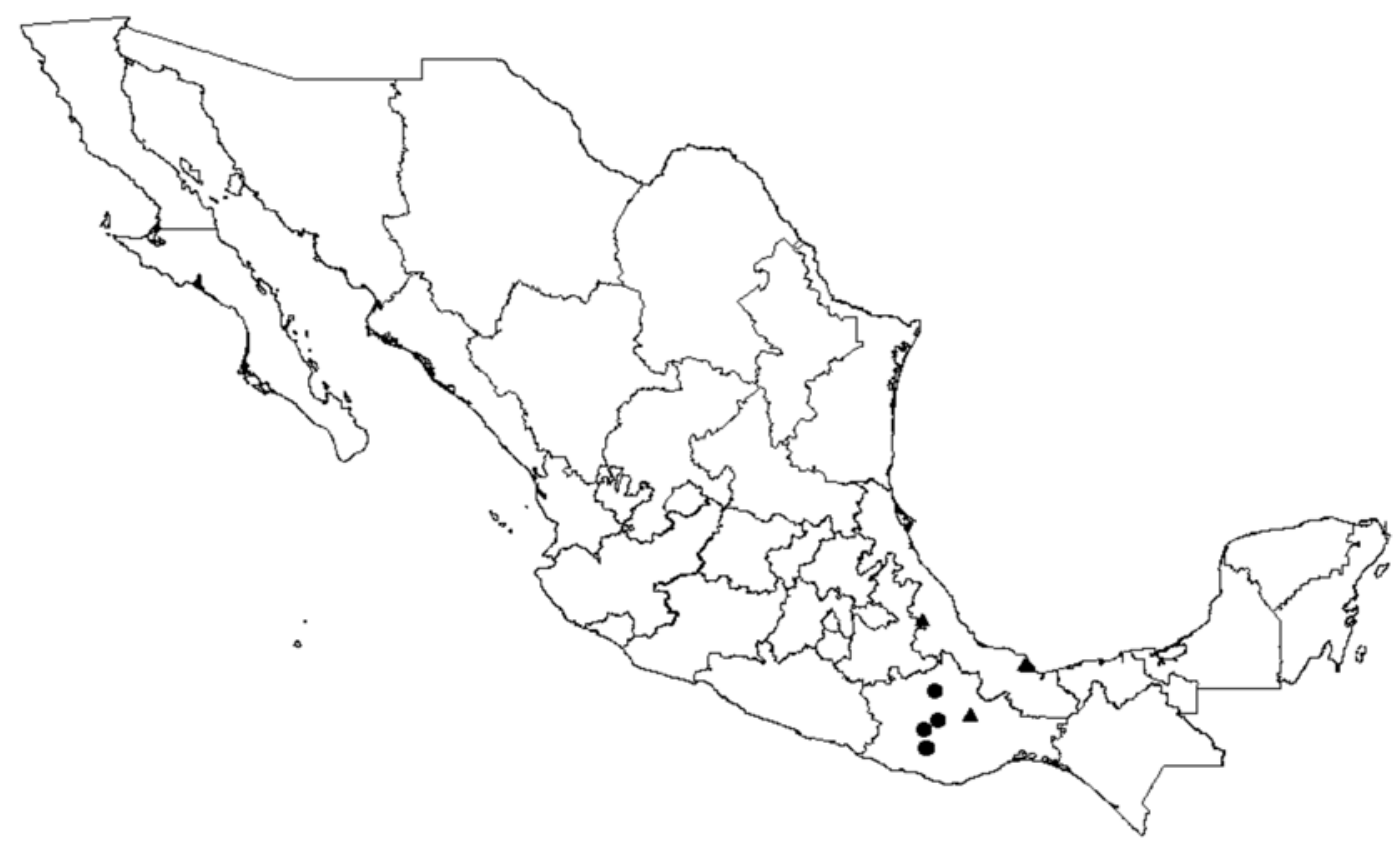

Figura 3. Mapa mostrando la localización de las poblaciones conocidas de Acianthera sotoana R.Solano (triangulos) y Stelis sotoarenasii R.Solano (círculos).

más pequeñas, las hojas más anchas y espatuladas, los segmentos florales ligeramente más grandes y el sépalo dorsal no está recurvado; su distribución se restringe a la Sierra Madre del Sur en Guerrero. Para la especie aquí propuesta como Stelis sotoarenasii se había usado el nombre inédito "Stelis zapotecana", con el cual fue citada por algunos autores recientemente (Soto-Arenas et al., 2007; Solano Gómez \& Soto Arenas, 2008; Solano Gómez et al., 2008).

Agradecimientos. A Eric Hágsater y dos revisores anónimos por sus cometarios para mejorar el manuscrito, a Salvador Acosta Castellanos por traducir las diagnosis e latín y a la Secretaría de Investigación y Posgrado del Instituto Politécnico Nacional por el apoyo financiero al proyecto "Orquídeas en riesgo del estado de Oaxaca" (clave 20090393).

\section{LITERATURA CITADA}

García-Cruz, J., L. Sánchez-Saldaña, R. Jiménez-Machorro \& R. Solano-Gómez. 2003. Familia Orchidaceae: Tribu Epidendreae. Pp. 1-173 in: J. Rzedowski \& G. Calderón de Rzedowski (eds.), Flora del Bajío y de Regiones Adyacentes, Fasciculo 119. Instituto de Ecología, A.C.-
CONACYT-CONABIO, Pátzcuaro, Michoacán.

Hágsater, E. \& G. Salazar-Chávez (eds.). 2003. Icon. Orchid. (Mexico) 1: Orchids of Mexico 1. Asociación Mexicana de Orquideología A.C., México, D.F.

Hágsater, E. \& M. Soto-Arenas (eds.). 1990. Icon. Orchid. (Mexico) 5-6: Orchids of Mexico 2-3. Herbario AMO. México, D.F.

Hágsater, E. \& M. Soto-Arenas (eds.). 2008. Icon. Orchid. (Mexico) 10: Orchids of Mexico 4. Herbario AMO. México, D.F.

López-Velázquez, G., R. Solano-Gómez \& M.A. PérezFarrera. 2007. Primer registro de Phloeophila (Orchidaceae: Pleurothallidinae) para la flora de México. Acta Bot. Mex. 78: 77-83.

Luer, C.A. 1991. New species of Pleurothallis. Lindleyana 6: 100-103.

Luer, C.A. 2004. New genera and new combinations in the Pleurothallidinae. Monogr. Syst. Bot. Missouri Bot. Gard. 95: 253-265.

Salazar-Chávez, G. \& M. Soto-Arenas. 1996. El género Lepanthes Sw. en México. Orquídea (Mex.) 14: 1-231.

Salazar-Chávez, G., J. Reyes, C. Brachet \& J. Pérez. 2006. Orquídeas y otras Plantas Nativas de la Cañada Cuicatlán, Oaxaca, México. Fundación para la Reserva de la Biosfera Cuicatlán A.C., Instituto de Biología 
UNAM, Comisión Federal de Electricidad y Sociedad Mexicana de Cactología A.C. México, D.F. 172 p.

Solano-Gómez, R. 1993. El género Stelis Sw. (Orchidaceae: Pleurothallidinae) en México. Orquídea (Mex.) 13: 1-112.

Solano-Gómez, R. 1999. Orchidaceae III. Stelis. Pp. 1-26 in: V. Sosa \& A. Gómez Pompa (eds.), Flora de Veracruz Fasciculo 113. Instituto de Ecología A.C. y Universidad de California, México.

Solano-Gómez, R. 2000. Additions to Stelis Sw. (Pleurothallidinae) from Mexico. Lindleyana 15: 46-52.

Solano-Gómez, R. \& G. Salazar-Chávez. 2007. A new species of Stelis (Orchidaceae, Pleurothallidinae) from Guerrero, Mexico. Rev. Mex. Biodiv. 78: 253-256.

Solano-Gómez, R., N. Alonso, K. Rosado, M. Aguilar \& R. García. 2008. Diversidad, distribución y estrategias para la conservación de las Pleurothallidinae (Orchidaceae) en Oaxaca. Bol. Soc. Bot. México 82: 41-52.
Solano-Gómez, R. \& M. Soto-Arenas. 2003. Acianthera johnsonii (Ames) Pridgeon \& M.W.Chase. Pl. 509 in: E. Hagsater \& M. Soto Arenas (eds.), Icon. Orchid. (Mexico) 5-6: Orchids of Mexico 2-3. Herbario AMO. México, D.F.

Solano-Gómez, R. \& M. Soto-Arenas. 2008. Stelis retusa (La Llave \& Lex.) Pridgeon \& M.W.Chase. Pl. 1098 in: E. Hagsater \& M. Soto Arenas (eds.), Icon. Orchid. (Mexico) 10: Orchids of Mexico 4. Herbario AMO. México, D.F.

Soto-Arenas, M. 1987. Una revisión de las especies mexicanas de Trichosalpinx subgénero Trichosalpinx. Orquídea (Mex.) 10: 247-277.

Soto-Arenas, M., E. Hágsater, R. Jiménez-Machorro \& R. Solano-Gómez. 2007. Orquídeas de México. Herbario AMO-Instituto Chinoín. A.C. y CIIDIR Unidad Oaxaca, Instituto Politécnico Nacional. Informe final SNIBCONABIO proyecto No. P107, México, D.F. 